\title{
An even unimodular 72-dimensional lattice of minimum 8.
}

\author{
Gabriele Nebe \\ Lehrstuhl D für Mathematik, RWTH Aachen University \\ 52056 Aachen, Germany \\ nebe@math.rwth-aachen.de
}

\begin{abstract}
An even unimodular 72-dimensional lattice $\Gamma$ having minimum 8 is constructed as a tensor product of the Barnes lattice and the Leech lattice over the ring of integers in the imaginary quadratic number field with discriminant -7 . The automorphism group of $\Gamma$ contains the absolutely irreducible rational matrix group $\left(\mathrm{PSL}_{2}(7) \times \mathrm{SL}_{2}(25)\right): 2$. Keywords: extremal even unimodular lattice, Hermitian tensor product. MSC: primary: 11H06, secondary: 11H31, 11H50, 11H55, 11H56, $11 \mathrm{H} 71$
\end{abstract}

\section{Introduction.}

In this paper a lattice $(L, Q)$ is always an even positive definite lattice, i.e. a free $\mathbb{Z}$-module $L$ equipped with a quadratic form $Q: L \rightarrow \mathbb{Z}$ such that the bilinear form

$$
(\cdot, \cdot): L \times L \rightarrow \mathbb{Z},(x, y):=Q(x+y)-Q(x)-Q(y)
$$

is positive definite on the real space $\mathbb{R} \otimes L$. The dual lattice is

$$
L^{\#}:=\{x \in \mathbb{R} \otimes L \mid(x, \ell) \in \mathbb{Z} \text { for all } \ell \in L\}
$$

and $L$ is called unimodular, if $L=L^{\#}$. The minimum of $L$ is twice the minimum of the quadratic form on the non-zero vectors of $L$

$$
\min (L)=\min \{(\ell, \ell) \mid 0 \neq \ell \in L\} .
$$

From the theory of modular forms it is known ([20], [10]) that the minimum of an even unimodular lattice of dimension $n$ is always $\leq 2\left\lfloor\frac{n}{24}\right\rfloor+2$. Lattices achieving this bound are called extremal. Of particular interest are extremal unimodular lattices in the so called "jump dimensions", these are the multiples of 24 . There are four even unimodular lattices known in the jump dimensions, the Leech lattice $\Lambda$, the unique even unimodular lattice in dimension 24 without roots, and three lattices called $P_{48 p}, P_{48 q}$, $P_{48 n}$, of dimension 48 which have minimum 6 [4], [14].

It was a long standing open problem whether there exists an extremal 72-dimensional unimodular lattice ([18, p. 151], [17, Section 3.4]). Many people tried to construct such a lattice, or to prove its non-existence. Most of these attempts are not documented, all constructed lattices contained vectors of norm 6. In [1] Christine Bachoc and I discovered two extremal lattices in dimension 80 of which we could prove extremality 
using a classical construction that we learned from [16]. Given a binary code $C \leq \mathbb{F}_{2}^{d}$ and an even lattice $L \leq \mathbb{R}^{n}$ of odd determinant together with a polarisation $L / 2 L=T_{1} \oplus T_{2}$ by isotropic subspaces the new lattice $\mathcal{L}\left(T_{1}, T_{2}, C\right)$ of dimension $d n$ is constructed as the preimage in $L^{d}$ of $T_{1} \otimes C \oplus T_{2} \otimes C^{\perp} \leq(L / 2 L)^{d}$. Inspired by [7] Christine and I used polarisations coming from Hermitian $\mathbb{Z}[\alpha]$-structures (where $\alpha^{2}-\alpha+2=0$ ) of $L$.

Bob Griess' article [5] analyses this construction for certain polarisations of the Leech lattice $\Lambda$ and $C=\langle(1,1,1)\rangle \leq \mathbb{F}_{2}^{3}$ for which he describes a strategy to prove extremality of the resulting lattice. This motivated me to try the nine $\mathbb{Z}[\alpha]$-structures of $\Lambda$ calculated in [8]. I computed the number of vectors of norm 6 in all nine 72 dimensional lattices using four different strategies: A combination of lattice reduction programs applied directly to the 72-dimensional lattice found vectors of norm 6 for all but one lattice. I then went on to compute the super offenders as described in [5, Section 4] and computed the number of norm 6 vectors in the lattices as given in Table 1 . Using the $\mathbb{Z}[\alpha]$ structure of $\Lambda$ this computation may be reduced to a computation within the set of minimal vectors of the Leech lattice. The result of these computations agreed with the ones applying the methods given in Section 4.

Using the explicit matrices for this extremal lattice $\Gamma$ and the action of the subgroup $G$ of $\operatorname{Aut}(\Gamma)$ as constructed in Section 2 Mark Watkins (personal communication) succeeded in listing representatives of all $G$-orbits of the vectors of norm 8 in $\Gamma$ using the method described in [19]. From the stored information one verifies that $\Gamma$ has $6,218,175,600$ minimal vectors which gives an independent proof of the extremality of $\Gamma$ (see also Theorem 3.5 for an explicit description of the kissing configuration of $\Gamma$ ).

\section{An Hermitian tensor product construction of $\Gamma$.}

Throughout the paper let $\alpha$ be a generator of the ring of integers $\mathbb{Z}[\alpha]$ in the imaginary quadratic number field of discriminant -7 , with $\alpha^{2}-\alpha+2=0$ and $\beta:=\bar{\alpha}=1-\alpha$ its complex conjugate. Then $\mathbb{Z}[\alpha]$ is a principal ideal domain and $(\alpha)$ and $(\beta)$ are the two maximal ideals of $\mathbb{Z}[\alpha]$ that contain 2 .

Let $(P, h)$ be an Hermitian $\mathbb{Z}[\alpha]$-lattice, so $P$ is a free $\mathbb{Z}[\alpha]$-module and $h: P \times P \rightarrow$ $\mathbb{Z}[\alpha]$ a positive definite Hermitian form. One example of such a lattice is the Barnes lattice

$$
P_{b}=\langle(\beta, \beta, 0),(0, \beta, \beta),(\alpha, \alpha, \alpha)\rangle=\langle(1,1, \alpha),(0, \beta, \beta),(0,0,2)\rangle \leq \mathbb{Z}[\alpha]^{3}
$$

with the half the standard Hermitian form

$$
h: P_{b} \times P_{b} \rightarrow \mathbb{Z}[\alpha], h\left(\left(a_{1}, a_{2}, a_{3}\right),\left(b_{1}, b_{2}, b_{3}\right)\right)=\frac{1}{2} \sum_{i=1}^{3} a_{i} \overline{b_{i}}
$$

Then $P_{b}$ is Hermitian unimodular, $P_{b}=P_{b}^{*}:=\left\{v \in \mathbb{Q} P_{b} \mid h(v, \ell) \in \mathbb{Z}[\alpha]\right.$ for all $\left.\ell \in P_{b}\right\}$. The automorphism group of the $\mathbb{Z}[\alpha]$-lattice $P_{b}$ is isomorphic to $\pm \mathrm{PSL}_{2}(7)$

$$
\operatorname{Aut}_{\mathbb{Z}[\alpha]}\left(P_{b}\right):=\left\{g \in \mathrm{GL}\left(P_{b}\right) \mid h(g v, g w)=h(v, w) \text { for all } v, w \in P_{b}\right\} \cong \pm \operatorname{PSL}_{2}(7) \text {. }
$$


From any such Hermitian $\mathbb{Z}[\alpha]$-lattice $(P, h)$ one obtains an even $\mathbb{Z}$-lattice

$$
L(P, h):=(L,(,)):=\left(P, \operatorname{trace}_{\mathbb{Z}[\alpha] / \mathbb{Z}} \circ h\right)
$$

by restricting scalars. Since $h$ is Hermitian $h(\ell, \ell) \in \mathbb{Z}=\mathbb{Z}[\alpha] \cap \mathbb{R}$ for all $\ell \in P$ and hence

$$
Q(\ell):=\frac{1}{2} \operatorname{trace} \mathbb{Z}[\alpha] / \mathbb{Z}(h(\ell, \ell))=h(\ell, \ell) \in \mathbb{Z} .
$$

The dual lattice of $L(P, h)$ is the product of $P^{*}$ with the different of $\mathbb{Z}[\alpha]$ :

$$
L(P, h)^{\#}:=\left\{v \in \mathbb{Q} P \mid \operatorname{trace}_{\mathbb{Z}[\alpha] / \mathbb{Z}}(h(\ell, v)) \in \mathbb{Z} \text { for all } \ell \in P\right\}=\frac{1}{\sqrt{-7}} P^{*} .
$$

Michael Hentschel [8] classified all Hermitian $\mathbb{Z}[\alpha]$-structures on the even unimodular $\mathbb{Z}$-lattices of dimension 24 using the Kneser neighbouring method ([1]) to generate the lattices and checking completeness with the mass formula. In particular there are exactly nine such $\mathbb{Z}[\alpha]$ structures $\left(P_{i}, h\right)(1 \leq i \leq 9)$ such that $\left(P_{i}, \frac{1}{7}\right.$ trace $\left.\mathbb{Z}[\alpha] / \mathbb{Z} \circ h\right) \cong \Lambda$ is the Leech lattice. The 36 -dimensional Hermitian $\mathbb{Z}[\alpha]$-lattice $R_{i}$ is defined as

$$
\left(R_{i}, h\right):=P_{b} \otimes_{\mathbb{Z}[\alpha]} P_{i} \text {, so } \operatorname{Aut}_{\mathbb{Z}[\alpha]}\left(R_{i}\right) \supseteq \operatorname{PSL}_{2}(7) \times \operatorname{Aut}_{\mathbb{Z}[\alpha]}\left(P_{i}\right) .
$$

Definition 2.1. For $1 \leq i \leq 9$ let $\left(\Gamma_{i},(),\right):=L\left(R_{i}, \frac{1}{7} h\right):=\left(R_{i}, \frac{1}{7}\right.$ trace $\left._{\mathbb{Z}[\alpha] / \mathbb{Z}} \circ h\right)$ where the quadratic form is $Q(\ell)=\frac{1}{14}$ trace $_{\mathbb{Z}}[\alpha] / \mathbb{Z}(h(\ell, \ell))$ for all $\ell \in \Gamma_{i}=R_{i}$.

All $\Gamma_{i}$ are even unimodular lattices of dimension 72 .

The table below lists these nine Hermitian structures of the Leech lattice. The first column gives the structure of the automorphism group $\operatorname{Aut}_{\mathbb{Z}[\alpha]}\left(P_{i}\right)$ followed by its order and then the number of vectors of norm 6 in the lattice $\Gamma_{i}$ (computed in Section 4 below).

Table 1

\begin{tabular}{|c|c|c|c|}
\hline & group & order & norm 6 vectors \\
\hline 1 & $\mathrm{SL}_{2}(25)$ & $2^{4} 3 \cdot 5^{2} 13$ & 0 \\
\hline 2 & $2 . A_{6} \times D_{8}$ & $2^{7} 3^{2} 5$ & $2 \cdot 20,160$ \\
\hline 3 & $\mathrm{SL}_{2}(13) .2$ & $2^{4} 3 \cdot 7 \cdot 13$ & $2 \cdot 52,416$ \\
\hline 4 & $\left(\mathrm{SL}_{2}(5) \times A_{5}\right) .2$ & $2^{6} 3^{2} 5^{2}$ & $2 \cdot 100,800$ \\
\hline 5 & $\left(\mathrm{SL}_{2}(5) \times A_{5}\right) .2$ & $2^{6} 3^{2} 5^{2}$ & $2 \cdot 100,800$ \\
\hline 6 & soluble & $2^{9} 3^{3}$ & $2 \cdot 177,408$ \\
\hline 7 & $\pm \mathrm{PSL}_{2}(7) \times\left(C_{7}: C_{3}\right)$ & $2^{4} 3^{2} 7^{2}$ & $2 \cdot 306,432$ \\
\hline 8 & $\mathrm{PSL}_{2}(7) \times 2 . A_{7}$ & $2^{7} 3^{3} 5 \cdot 7^{2}$ & $2 \cdot 504,000$ \\
\hline 9 & $2 . J_{2} .2$ & $2^{9} 3^{3} 5^{2} 7$ & $2 \cdot 1,209,600$ \\
\hline
\end{tabular}

Remark 2.2. (a) The groups number 1, 3, 4,5, and 9 are maximal finite quaternionic matrix groups with endomorphism algebra the definite quaternion algebra with center $\mathbb{Q}$ and discriminant $5^{2}(1,4,5,9)$ resp. $13^{2}$ (group number 3$)$ (see [15]). For the group number 4 resp. 5, the endomorphism ring of the lattice is not the maximal order.

(b) The group number 8 is a maximal finite symplectic matrix group over $\mathbb{Q}[\alpha]$ as defined in [9], it is globally irreducible in the sense of [6].

(c) The groups number 2 and 7 are reducible. 
The Hermitian structures number 4 and 5 are just Galois conjugate to each other, whereas all the others are Galois invariant. For these seven lattices the automorphism group of the $\mathbb{Z}$-lattice $\Gamma_{i}$ hence contains an extension of $\operatorname{Aut}_{\mathbb{Z}[\alpha]}\left(R_{i}\right)$ by the Galois automorphism. For the extremal lattice $\Gamma:=\Gamma_{1}$ this is a split extension.

Theorem 2.3. The lattice $\Gamma$ is an extremal even unimodular lattice of dimension 72 . Its automorphism group $\operatorname{Aut}(\Gamma)$ contains the subgroup $\mathcal{U}:=\left(\mathrm{PSL}_{2}(7) \times \mathrm{SL}_{2}(25)\right): 2$.

Two proofs that the minimum of $\Gamma$ is 8 are given below.

Remark 2.4. The natural $\mathbb{C} \mathcal{U}$-module $\mathbb{C} \otimes \Gamma$ contains no $\mathcal{U}$-invariant submodules, so $\operatorname{Aut}(\Gamma)$ is an absolutely irreducible subgroup of $\mathrm{GL}_{72}(\mathbb{Q})$. In fact $\mathcal{U}$ is almost a globally irreducible representation in the sense of [6]. More precisely $\mathbb{F}_{p} \otimes \Gamma$ is also absolutely irreducible except for $p=5$ and $p=7$, where the module has a unique non-trivial submodule, which is of dimension 36. For both primes $p=5$ and $p=7$ there is an element $x_{p} \in N_{\mathrm{GL}_{72}(\mathbb{Q})}(\mathcal{U})$, the rational normalizer of $\mathcal{U}$ mapping $\Gamma$ to the unique sublattice of index $p^{36}$, which is therefore isometric to $(\Gamma, p Q)$ (see [12]). Therefore Aut $(\Gamma)$ is a maximal finite subgroup of $\mathrm{GL}_{72}(\mathbb{Q})$.

Remark 2.5. Since Aut $(\Gamma)$ contains an element of order 91 the lattice $\Gamma$ is an ideal lattice in the cyclotomic field $\mathbb{Q}[\exp (2 \pi i / 91)]$ in the sense of [2]. It would be interesting to determine the ideal class of this lattice.

\subsection{An elementary linear algebra construction.}

This section just repeats the construction above in elementary linear algebra (understood by computer algebra systems).

Let $\left(b_{1}, \ldots, b_{24}\right)$ be a $\mathbb{Z}$-basis of the Leech lattice $\Lambda$ and $F:=\left(\left(b_{i}, b_{j}\right)\right) \in \mathbb{Z}_{\text {sym }}^{24 \times 24}$ denote its Gram matrix. Then an Hermitian structure over $\mathbb{Z}[\alpha]$ is given by a matrix $A \in \mathbb{Z}^{24 \times 24}$ such that $A F A^{t r}=2 F$ and the $F$-adjoint $F A^{t r} F^{-1}=1-A=: B$. Mapping $\alpha$ to the right multiplication by $A$ then defines the Hermitian $\mathbb{Z}[\alpha]$-structure on the $\mathbb{Z}$-lattice $\Lambda$.

That there are exactly nine such $\mathbb{Z}[\alpha]$ structures of the Leech lattice means that there are nine such matrices $A_{1}, \ldots, A_{9}$ up to conjugation under the automorphism group of $\Lambda$.

For any of these nine structures the even unimodular lattice $\Gamma_{i}$ of dimension 72 is constructed as a sublattice of $\Lambda \perp \Lambda \perp \Lambda$ with $\operatorname{Gram}$ matrix $\frac{1}{2} \operatorname{diag}(F, F, F)$ generated by the rows of the block matrix

$$
\left(\begin{array}{ccc}
A_{i} & A_{i} & A_{i} \\
B_{i} & B_{i} & 0 \\
0 & B_{i} & B_{i}
\end{array}\right) \text { or equivalently }\left(\begin{array}{ccc}
1 & 1 & A_{i} \\
0 & B_{i} & B_{i} \\
0 & 0 & 2
\end{array}\right)=: T_{i}
$$

If $U$ denotes the subgroup of $\mathrm{GL}_{72}(\mathbb{Z})$ obtained by replacing $\alpha$ by $A_{i}$ in the group $\operatorname{Aut}_{\mathbb{Z}[\alpha]}\left(P_{b}\right) \leq \mathrm{GL}_{3}(\mathbb{Z}[\alpha])$ isomorphic to $\operatorname{PSL}_{2}(7)$ then $\operatorname{Aut}\left(\Gamma_{i}\right)$ contains the matrix group

$$
\left\langle\left\{\operatorname{diag}(g, g, g) \mid g \in \operatorname{Aut}(\Lambda), g A_{i}=A_{i} g\right\} \cup U\right\rangle \cong \operatorname{Aut}_{\mathbb{Z}[\alpha]}\left(P_{i}\right) \times \operatorname{PSL}_{2}(7) .
$$


A matrix for the additional Galois automorphism with respect to the basis given by $T_{i}$ above can be constructed from an isometry

$$
Y_{i} \in \mathrm{GL}_{24}(\mathbb{Z}), \quad Y_{i} F Y_{i}^{\mathrm{tr}}=F, \quad Y_{i} A_{i} Y_{i}^{-1}=B_{i}
$$

(this only exists for $i \neq 4,5$ ) as the block matrix

$$
\left(\begin{array}{ccc}
Y_{i} & -Y_{i} & A_{i} Y_{i} \\
0 & -B_{i} Y_{i} & Y_{i} \\
-A Y_{i} & 0 & Y_{i}
\end{array}\right)
$$

The shape of the matrix was obtained from an isometry between $\left(P_{b}, h\right)$ and $\left(P_{b}, \bar{h}\right)$.

\subsection{A classical coding theory construction.}

The lattices $\Gamma_{i}$ can be obtained using a special case of a classical construction with codes: If $(L, Q)$ is an even unimodular lattice, then $L / 2 L$ becomes a non-degenerate quadratic space over $\mathbb{F}_{2}$ with quadratic form $q(\ell+2 L):=Q(\ell)+2 \mathbb{Z}$. This has Witt defect 0 , so there are totally isotropic subspaces $U, V \leq(L / 2 L, q)$ such that $L / 2 L=U \oplus V$. Let $2 L \leq M, N \leq L$ denote the preimages of $U, V$, respectively. Then $\left(M, \frac{1}{2} Q\right)$ and $\left(N, \frac{1}{2} Q\right)$ are again even unimodular lattices.

Definition 2.6. ([5], [16, Construction I]) Given such a polarisation $(M, N)$ of the even unimodular lattice $(L, Q)$ and some $k \in \mathbb{N}$ let

$L(M, N, k):=\left\{\left(x_{1}+y, x_{2}+y, \ldots, x_{k}+y\right) \mid y \in N, x_{1}, \ldots, x_{k} \in M\right.$ and $\left.x_{1}+\ldots+x_{k} \in M \cap N\right\}$.

Then the lattice $(L(M, N, k), \tilde{Q})$ is an even unimodular lattice ([16, Proposition]) where

$$
\tilde{Q}\left(x_{1}+y, x_{2}+y, \ldots, x_{k}+y\right):=\frac{1}{2} \sum_{i=1}^{k} Q\left(x_{i}+y\right) .
$$

From the explicit basis of the Barnes lattice given in Section 2 one immediately sees the following.

Remark 2.7. Assume that the lattice $L$ has an Hermitian structure over $\mathbb{Z}[\alpha]$ as defined in Section 2. Then $M:=\alpha L$ and $N:=\beta L$ defines a polarisation of $L$ such that $L(M, N, 3) \cong P_{b} \otimes_{\mathbb{Z}[\alpha]} L$.

Remark 2.8. ([5, Theorem 4.10]) Assume that $L=\Lambda \cong\left(M, \frac{1}{2} Q\right) \cong\left(N, \frac{1}{2} Q\right)$ is the Leech lattice. Then $L(M, N, 3)$ has minimum 6 or 8 . The vectors of norm 6 in $L(M, N, 3)$ are of the form $(w+x, w+y, w+z)$ with $w \in N, x, y, z \in M, x+y+z \in 2 \Lambda$ and $Q(w+x)=Q(w+y)=Q(w+z)=2$.

The next section analyses this construction in particular for this situation. Using properties of the Leech lattice a description of the vectors of norm 6 and 8 in $L(M, N, 3)$, in particular of the kissing configuration of the new extremal lattice, is obtained. I thank Noam Elkies for proposing this interesting question. 


\section{The vectors of norm 6 and 8 in $L(M, N, 3)$.}

Let $(\Lambda, Q)$ be the Leech lattice, and fix a polarisation $(M, N)$ of $\Lambda$ such that $\left(M, \frac{1}{2} Q\right) \cong$ $\left(N, \frac{1}{2} Q\right) \cong \Lambda$.

The following property of the Leech lattice is well known.

Lemma 3.1. The nontrivial classes of $\Lambda / 2 \Lambda$ are represented by vectors $v \in \Lambda$ of norm $(v, v)=4,6$ and 8. In particular all classes of $M / 2 \Lambda$ and $N / 2 \Lambda$ are represented by vectors of norm 8 . If $K=v+2 \Lambda$ contains a vector of norm 8 , then $\{k \in K \mid(k, k) \leq$ $8\}=\left\{ \pm k_{1}, \ldots, \pm k_{24}\right\}$ with $\left(k_{i}, k_{j}\right)=8 \delta_{i j}$. If $K=v+2 \Lambda$ contains a vector $v$ of norm 4 or 6 then $\{k \in K \mid(k, k) \leq 8\}=\{ \pm v\}$.

Proof. Let $(v, v)=4$ or $(v, v)=6$ and assume that there is some $\pm v \neq k \in v+2 \Lambda$ such that $(k, k) \leq 8$. Then one of $v \pm k \in 2 \Lambda$ has norm $\leq 6+8<16=\min (2 \Lambda)$ which is a contradiction. Similarly one sees that for $(v, v)=8$ the vectors of norm 8 in $v+2 \Lambda$ form a frame. Now

$$
\frac{\left|\Lambda_{4}\right|}{2}+\frac{\left|\Lambda_{6}\right|}{2}+\frac{\left|\Lambda_{8}\right|}{48}=2^{24}-1
$$

so all nonzero classes of $\Lambda / 2 \Lambda$ are represented by vectors of norm $\leq 8$.

Proposition 3.2. Fix some $w \notin M$ with $(w, w)=8$. Then $W_{3}(w):=\{x \in M \mid$ $Q(x+w)=3\}$ has cardinality $2 \cdot 2048$.

Proof. If $x \in M$ such that $Q(w+x)=3$ then the class $K_{x}:=x+2 \Lambda$ is not perpendicular to $K_{w}$. Since $w \notin M$ there are $2^{11}=2048$ classes $x+2 \Lambda \in M / 2 \Lambda$ that are not perpendicular to $K_{w} \in N / 2 \Lambda$ by the polarisation property. So there are 2048 possibilities for the class $K_{w+x}=K_{x}+K_{w}=(x+w)+2 \Lambda$. Such a class is necessarily anisotropic and therefore contains exactly 2 vectors of norm 6 by Lemma 3.1 .

Remark 3.3. Let $\tilde{X}:=\{m \in M \mid(m, m)=8\}$ denote the set of minimal vectors in $M$. Then $\tilde{X}$ is a spherical 11-design. For any $w \in N$ with $(w, w)=8$ and $i \in \mathbb{Z}_{\geq 0}$ let

$$
n_{i}:=|\{x \in \tilde{X} \mid(x, w)= \pm i\}|
$$

Then $n_{i}=0$ if $i \geq 7$,

$$
\sum_{i \text { odd }} n_{i}=2048 \cdot 48 \text { and } \sum_{i \text { even }} n_{i}=2047 \cdot 48 .
$$

This allows to compute all $n_{i}$ using the 11-design properties of $\tilde{X}$ (see for instance [21] for a description of this method):

\begin{tabular}{|c|c|c|c|c|c|c|c|}
\hline$i$ & 0 & 1 & 2 & 3 & 4 & 5 & 6 \\
\hline$n_{i}$ & 46488 & 78848 & 47216 & 18944 & 4536 & 512 & 16 \\
\hline
\end{tabular}

In particular $S(w):=\{x \in \tilde{X} \mid(x, w)=-6\}$ contains exactly 8 elements. The only property we need is that this set is not empty. 
Proposition 3.4. Fix some $w \in N$ with $(w, w)=8$. Then $W_{2}(w):=\{x \in M \mid$ $Q(x+w)=2\}$ has cardinality $2 \cdot 24$. The set $\left\{x+w \mid x \in W_{2}(w)\right\}$ is the rescaled root system $24 \mathbb{A}_{1}$.

Proof. Since $w \notin M$ and $M / 2 \Lambda$ is maximal isotropic there is some $m \in M$ such that $(m, w)$ is odd and in particular $M_{w}:=\{m \in M \mid(m, w)$ even $\}$ is a sublattice of index 2 in $M$ and $M^{w}:=\left\langle M_{w}, w\right\rangle=M_{w} \dot{\cup} w+M_{w}$ is a neighbor of $M$ in the sense of [11] and again isometric to a rescaled even unimodular lattice. Since $M$ has no roots the root system of $M^{w}$ is either empty or $24 \mathbb{A}_{1}$ (rescaled to have minimum 4). Remark 3.3 shows that this lattice contains at least 16 roots, so there are 24 pairs of orthogonal vectors of norm 4 in $M^{w}$ giving rise to the $x+w$ with $x \in W_{2}(w)$.

Theorem 3.5. The vectors of norm 6 and 8 in $L(M, N, 3)$ are of the form $(w+x, w+$ $y, w+z)$ with $w \in M, x, y, z \in N, x+y+z \in 2 \Lambda$ such that $Q(w+x)+Q(w+y)+Q(w+$ $z)=6$ or 8 . For norm 6 the only possibility is $Q(w+x)=Q(w+y)=Q(w+z)=2$. Let $b_{6}$ denote the number of such vectors. For norm 8 one has the possible types

(a) $(8,0,0)$ with $196560 \cdot 3$ vectors.

(b) $(4,4,0)$ with $196560 \cdot 48 \cdot 3$ vectors.

(c) $(3,3,2)$ with $4095 \cdot 48 \cdot 2048 \cdot 2 \cdot 2 \cdot 3$ vectors.

(d) $(4,2,2)$ with $4095 \cdot 48 \cdot 48 \cdot 48 \cdot 3-72 b_{6}$ vectors.

Proof. Clearly these are the only possibilities for vectors of norm 6 or 8 in $L(M, N, 3)$. The vectors of type $(8,0,0)$ correspond to the minimal vectors in the sublattice $2 \Lambda \perp$ $2 \Lambda \perp 2 \Lambda$. The vectors of type $(4,4,0)$ are of the form $(x, y, 0)$ with $x, y \in M,(x, x)=$ $(y, y)=8$ such that $x+2 \Lambda=y+2 \Lambda$, so one has 196560 possibilities for $x$ and for each such $x$ one may choose all 48 minimal vectors $y \in x+2 \Lambda$. The additional factor 3 counts the possible permutations $(x, y, 0),(x, 0, y)$, and $(0, x, y)$.

To see (c) we first note that by Lemma 3.1 all anisotropic classes in $\Lambda / 2 \Lambda$ are represented by vectors of norm 6 . For a fixed representative $w$ of one of the 4095 classes of $N / 2 \Lambda$ of norm $(w, w)=8$ we have to run through all $z \in W_{2}(w)$ and $y \in W_{3}(w)$. Then the condition that $x+y+z \in 2 \Lambda$ means that $w+x+2 \Lambda=y+z+2 \Lambda$, so $w+x$ is one of the 2 vectors of norm 6 in this anisotropic class.

The last set is the set of vectors of Type $(4,2,2)$. Again, fixing some $w$ as above the elements $y$ and $z$ are in $W_{2}(w)$. So we have 48 possibilities of each of them. Then $w+x \in w+y+z+2 \Lambda$ is in an isotropic class of $\Lambda / 2 \Lambda$. This class is either of minimum 4 and then we have 2 vectors in this class resulting in a vector of norm 6 in $L(M, N, 3)$ or this class is of minimum 8 and there are 48 possibilities for $w+x$ such that $Q(w+x)=4$. 


\section{Two proofs that the minimum of $\Gamma$ is 8}

\subsection{Counting the norm 6 vectors in $L(M, N, 3)$}

Let $W:=\left\{w_{1}, \ldots, w_{4095}\right\}$ denote a fixed set of representatives of the classes in $N / 2 \Lambda$ consisting of vectors of norm 8 . The vectors of norm 6 in $\Gamma$ are of the form $(w+x, w+$ $y, w+z)$ where $w \in W, x, y, z \in W_{2}(w)$ and $x+y+z \in 2 \Lambda$. The set $W_{2}(w)$ may be computed as the set $W_{2}(w)=\left\{s-w \mid s \in M^{w},(s, s)=4\right\}$ as described in Proposition 3.4 .

Remark 4.1. To count the vectors of norm 6 and 8 in $\Gamma_{i}$ let $w \in W$ run through representatives of the $\operatorname{Aut}_{\mathbb{Z}[\alpha]}\left(P_{i}\right)$-orbits on $N / 2 \Lambda$.

For each such $w$ compute the set $W_{2}(w)=\left\{s-w \mid s \in M^{w},(s, s)=4\right\}$. Then run through the pairs $(x, y) \in W_{2}(w)^{2}$ and compute the vectors of norm 4 in the lattice $\langle 2 \Lambda, w+x+y\rangle$. This lattice either has two vectors of norm 4 contributing to the vectors of norm 6 in $\Gamma_{i}$ or it has minimum 8 and then it contains 48 vectors of norm 8 contributing to the vectors of norm 8 in $\Gamma_{i}$.

Using this method I found the number of vectors of norm 6 in $\Gamma_{i}$ as given in Table 1.

Remark 4.2. As proposed by Bob Griess in [5, Lemma B.3] I checked all pairs of fourvolutions $f, g \in \operatorname{Aut}(\Lambda)$ such that $x:=f g$ is an element of odd prime order $p$ with irreducible minimal polynomial. Then $p=3,5,7,13$ and the conjugacy class of $x$ in 2.Co $\mathrm{O}_{1}$ is unique. To enumerate all such pairs, I computed the normaliser $N$ of $\langle x\rangle$ in 2.Co $o_{1}$ and went through all conjugacy classes of elements $f$ of $N$ such that $f^{2}=-1$. For each such $f$ that satisfies $x^{f}=x^{-1} I$ put $g:=f^{-1} x$. The centraliser in 2.Co $o_{1}$ acts on the situation. All six lattices $L(\Lambda(f-1), \Lambda(g-1), 3)$ contain vectors of norm 6 .

Table 2

\begin{tabular}{|c|c|c|}
\hline$x$ & centraliser order & norm 6 vectors \\
\hline 3 & $2^{8} 3^{3} 5^{2} 7$ & $2 \cdot 1,209,600$ \\
$5 a$ & $2^{8} 3^{3} 5^{2} 7$ & $2 \cdot 1,209,600$ \\
$5 b$ & $2^{8} 3 \cdot 5$ & $2 \cdot 103680$ \\
7 & $2^{3} 3 \cdot 5$ & $2 \cdot 11520$ \\
13 & $2^{3} 3$ & $2 \cdot 57600$ \\
\hline
\end{tabular}

\subsection{Using orthogonal decomposition $(24,48)$.}

The idea (see [13]) is to embed the lattice $\Gamma_{i}$ into an orthogonally decomposable lattice $I_{1} \perp I_{2}$ such that the minimal vectors of $I_{1}$ and the minimum of sublattices of $I_{2}$ can be computed. The even unimodular lattice $\Gamma_{i}$ has basis matrix $T_{i}$ given in Section 2 with respect to the Gram matrix $\frac{1}{2} \operatorname{diag}(F, F, F)$. Let $\pi$ denote the orthogonal projection onto the first 24 components, $K_{1}:=\operatorname{ker}(1-\pi) \subset I_{1}:=\operatorname{im}(\pi)=K_{1}^{\#}$, and $K_{2}:=$ $\operatorname{ker}(\pi) \subset I_{2}:=\operatorname{im}(1-\pi)=K_{2}^{\#}$. Then

$$
K_{1} \perp K_{2} \subset \Gamma_{i} \subset I_{1} \perp I_{2} .
$$

So the even unimodular lattice $\Gamma_{i}$ contains the sublattice $K_{1} \perp K_{2}$ of index $2^{24}$ and is contained in $I_{1} \perp I_{2}$ also of index $2^{24}$. Moreover $I_{1} \cong \frac{1}{\sqrt{2}} \Lambda$ is isometric to the Leech 
lattice scaled to have minimum 2 and $I_{2}$ is a non integral lattice of dimension 48, with $(\ell, \ell) \in \mathbb{Z}$ for all $\ell \in I_{2}$. A computer calculation shows that $\min \left(I_{2}\right)=4, K_{1} \cong \sqrt{2} \Lambda$ has minimum 8 and also the 48-dimensional sublattice $K_{2}$ of $\Gamma_{i}$ has minimum 8.

Proposition 4.3. The vectors of norm 6 in $\Gamma_{i}$ are of the form $x+y$ with $x \in I_{1}$ of norm 2 and $y \in I_{2}$ of norm 4.

For all $i=1, \ldots, 9 \mathrm{I}$ computed representatives $v$ of the orbits of $\operatorname{Aut}_{\mathbb{Z}[\alpha]}\left(P_{i}\right)$ on the minimal vectors of $I_{1}$. For each $v$ there is some $w \in I_{2}$ such that $v+w \in \Gamma_{i}$, moreover $w$ is unique modulo $K_{2}$. So it remains to check that the minimum of the 48 dimensional lattice

$$
I(w):=\left\langle K_{2}, w\right\rangle \leq I_{2}
$$

is $\geq 6$. This is done by enumerating all vectors of norm 4 in this lattice.

Remark 4.4. For $\Gamma_{2}, \ldots, \Gamma_{9}$ the lattice $I(w)$ contains vectors of norm 4 for some $w$, summing up to the number of vectors of norm 6 in $\Gamma_{i}$ given in Table 1 . Only for the lattice $\Gamma=\Gamma_{1}$ the representatives $w$ of all 15 orbits of $\mathrm{SL}_{2}(25)$ provide lattices $I(w)$ of minimum $>4$.

\section{References}

[1] Christine Bachoc, Gabriele Nebe, Extremal lattices of minimum 8 related to the Mathieu group $M_{22}$. J. reine angew. Math. 494 (1998) 155-171.

[2] Eva Bayer-Fluckiger, Ideal lattices. Proc. of the conference in honor of Alan Baker, Number Theory and Diophantine Geometry, Cambridge Univ. Press (2002) 168184 .

[3] W. Bosma, J. Cannon, C. Playoust, The Magma algebra system. I. The user language. J. Symbolic Comput., 24(3-4):235-265, 1997

[4] J.H. Conway, N.J.A. Sloane, Sphere packings, lattices and groups. Springer Grundlehren 290, 1993.

[5] Robert L. Griess Jr., Rank 72 high minimum norm lattices. J. Number Theory 130 (2010) 1512-1519.

[6] B. Gross, Group representations and lattices. J. Amer. Math. Soc. 3 (1990) 929960.

[7] B. Gross, N. Elkies, The exceptional cone and the Leech lattice, Int. Math. Res. Notices 14 (1996) 665-698.

[8] M. Hentschel, On Hermitian theta series and modular forms. Thesis RWTH Aachen 2009.

[9] M. Kirschmer, Finite symplectic matrix groups. Thesis RWTH Aachen 2009, Exp. Math. (to appear) 
[10] C. L. Mallows, A. M. Odlyzko and N. J. A. Sloane, Upper Bounds for Modular Forms, Lattices, and Codes. J. Algebra 36 (1975) 68-76.

[11] M. Kneser, Klassenzahlen definiter quadratischer Formen. Archiv der Math. 8 (1957) 241-250.

[12] G. Nebe, The normaliser action and strongly modular lattices. L'Ens. Math., 43 (1997) 67-76

[13] G. Nebe, Construction and investigation of lattices with matrix groups. in Integral Quadratic Forms and Lattices edited by Myung-Hwan Kim, J. S. Hsia, Y. Kitaoka, R. Schulze-Pillot, Contemporary Mathematics 249 (1999), 205-220.

[14] G. Nebe, Some cyclo-quaternionic lattices, J. Alg. 199 (1998) 472-498.

[15] G. Nebe, Finite quaternionic matrix groups. Represent. Theory 2 (1998) 106-223.

[16] H.-G. Quebbemann, A construction of integral lattices. Mathematika, 31 (1984) 137-140.

[17] R. Scharlau, R. Schulze-Pillot, Extremal lattices. Algorithmic algebra and number theory (Heidelberg, 1997), Springer (1999) 139-170.

[18] N. J. A. Sloane, Binary Codes, Lattices, and Sphere-Packings. in Combinatorial Surveys: Proceedings of the Sixth British Combinatorial Conference, P. J. Cameron (editor), Academic Press, NY (1977) 117-164.

[19] D. Stehlé, M. Watkins, On the Extremality of an 80-Dimensional Lattice. ANTS 2010: 340-356.

[20] C.L. Siegel, Berechnung von Zetafunktionen an ganzzahligen Stellen. Göttinger Nach. 10 (1969) 87-102.

[21] B.B. Venkov, Réseaux et designs sphériques, Monogr. Ens. Math. vol. 37, (2001) $10-86$ 